\title{
Psycho-emotional disorders as incoming risk factors for myocardial infarction with non-obstructive coronary arteries
}

\author{
Javier López Pais ${ }^{1}$, Bárbara Izquierdo Coronel $^{1}$, David Galán Gil ${ }^{1}$, \\ Maria Jesús Espinosa Pascual ${ }^{1}$, Carlos Gustavo Martinez Peredo', \\ Paula Awamleh Garcia ${ }^{1}$, Juan Górriz Magaña ${ }^{1}$, Rebeca Mata Caballero ${ }^{1}$, \\ Alfonso Fraile Sanz ${ }^{1}$, Javier Muñiz ${ }^{2}$, Joaquín J. Alonso Martín ${ }^{1}$ \\ ${ }^{1}$ Cardiology Department, Hospital Universitario de Getafe, Spain \\ ${ }^{2}$ Instituto Universitario de Ciencias de la Salud, Universidad de A Coruña, Spain
}

\begin{abstract}
Background: There is an emerging field underlying the myocardial infarction (MI) with non-obstructive coronary arteries (MINOCA). The aim of this study was to evaluate the impact of psycho-emotional disorders and social habits in MINOCA patients.

Methods: The study included 95 consecutive patients diagnosed of MINOCA and 178 patients with MI and obstructive lesions. MINOCA patients were included when they fulfilled the three main criteria: accomplishment of the Third Universal Definition of Myocardial Infarction, absence of obstructive coronary arteries and no clinically overt specific cause for the acute presentation.

Results: MINOCA patients had a higher frequency of previous psychiatric illnesses than the obstructive coronary arteries group $(29.7 \%$ vs. $12.9 \%, p=0.001)$. MINOCA patients recognized emotional stress in $75.7 \%$ of the cases, while only $32.1 \%$ of the obstructive related group did $(p<0.001)$. The relationship remained after excluding takotsubo syndrome from the analysis (26 cases, 27.4\%): psychiatric diseases (27.9\% vs. $12.9 \%, p<0.01)$ and recognition of emotional stress $(70.8 \%$ vs. $32.1 \%, p<0.001)$. Social habits which could act as stress modulating showed no significant relation with MINOCA.

Conclusions: Psycho-emotional disorders are related to MINOCA and they could act as risk factor. This relationship is maintained after excluding takotsubo from the analysis. (Cardiol J 2018; 25, 1:24-31)
\end{abstract}

Key words: psycho-emotional disorders, stressful habits, myocardial infarction with non-obstructive coronary arteries, risk factors, acute coronary syndrome

\section{Introduction}

Myocardial infarction (MI) with non-obstructive coronary arteries (MINOCA) has become an issue of growing interest in cardiology over the last few years $[1,2]$. The majority of acute MI are related to complications of coronary atherosclerosis
[3]. Nevertheless, there is an important subgroup of patients with MI who undergo coronary angiogram and have no significant obstructive coronary artery disease. Large acute MI registries showed [4-6] an incidence of between $6 \%$ and $13 \%$ of patients. In the Spanish population, the DIOCLES study [7], it was observed that $5 \%$ of patients with

Address for correspondence: Javier López Pais, MD, Cardiology Department, Hospital Universitario de Getafe, Carretera de Toledo s/n KM 12.5, Postal Code 28905, Tlf: 916839360, Spain, e-mail: javierlopezpais@gmail.com 
ST-elevation MI (STEMI), $15.4 \%$ of the non-STEMI patients and $18 \%$ of the unclassified ones had no significant angiographic lesions.

There are scant data about the underlying cause of MINOCA and if there are any risk factors that should especially be taken into account to prevent an event in this determined group of patients. One of the most important limitations in the MINOCA study is the absence of clear definitions. There is no homogeneity in the existing literature about this since each group has used different inclusion criteria [8-12]. That fact could have changed after the European Society of Cardiology (ESC) 2016 Position Paper on MINOCA, where MINOCA is defined as a working diagnosis [6] given to a conundrum of different pathologies [9].

The absence of obstructive lesions leads to a wide field of other causes for the acute coronary syndrome to be taken into account: plaque disruption, coronary artery spasm, coronary thromboembolism, coronary dissection, takotsubo cardiomyopathy and type- 2 acute MI (necrosis due to a supply-demand mismatch). It is very important to delve into exhaustive diagnosis tests in these patients once the coronariography has been performed, this has been reflected in the latest European Clinical Guidelines [13], which introduced MINOCA for the first time, highlighting the need of determining the etiology for the best-targeted treatment in each case. Myocarditis is a different entity and should not be included in the working diagnosis unless it is not suspected from the beginning and turns out to be a final discovery after the etiological study is completed.

In the last few years, several studies trying to define MINOCA patient characteristics have been developed, with special mention to the systematic review of Pasupathy et al. [8]. In general terms, they tend to be younger patients, with fewer traditional risk factors, have a better prognosis and also have a probable trigger event behind the initiation of symptoms [14]. Also, psycho-emotional disorders could play a special role and may be an interesting medical target for both the prevention and the complete healing in some MINOCA patients [15].

Recent research showed that psychiatric disease is associated with takotsubo syndrome, one of the most prevalent mechanisms underlying MINOCA [16]. The precise mechanism which could explain this connection has not yet been elucidated, although a few theories have been described. The most extended one relates to the increase in catecholamines [16]. Whether the rest of non-takotsubo individuals and MINOCA patients have the same emotional trigger has not yet been studied.

The primary aim of this study is to analyse the association between psycho-emotional disorders and social habits which could lead to emotional dysfunctions with MINOCA patients. As just mentioned, recent research described the relationship between takotsubo and psychiatric diseases [16]. As hypothesized herein, the relationship between psychiatric diseases and psycho-emotional disorders could be inferred to all MINOCA patients, the secondary objective of this study is to analyze the subgroup of non-takotsubo MINOCA (N-TT MINOCA) patients on its own.

\section{Methods}

\section{Study population}

This study is a subproject of the IMACORN Registry, which has been in development at the Hospital Universitario de Getafe since 2015 in order to find out the epidemiology, clinical features and the physiopathology of MINOCA.

One hundred and two consecutive patients diagnosed with MINOCA were included from March 2015 until July 2017 in the Hospital Universitario Getafe which is a reference centre for a total health population of 220,000 individuals. Patients were included in the registry on the basis of ESC three criteria [6] as follows: First, MI criteria of the Third Universal Definition of Myocardial Infarction [17], which means positive cardiac biomarker and corroborative clinical evidence of infarction (symptoms of ischemia, new or presumed new significant ST-T changes or new left bundle branch block, development of pathological $\mathrm{Q}$ waves, imaging evidence of new loss of viable myocardium or evident intracoronary thrombus). Second, the definition of non-obstructive coronary arteries on angiography, consistent with contemporary angiography guidelines [18]: No coronary artery stenosis $\geq 50 \%$ in any potential infarct-related artery. Third, no clinically overt specific cause for the acute presentation. In cases of suspicion of pulmonary embolism, D-dimer was used to rule it out, and if positive, computed tomography was performed to discard it. Takotsubo syndrome was diagnosed based on Mayo Clinic Criteria [19]. Myocarditis cases were only included in the working diagnosis of MINOCA when their initial clinical presentation suggested an MI rather than myocarditis. All cases were reviewed by 2 cardiologists who were not involved in clinical management of the patients. After that, 3 were excluded for not 
fulfilling the criteria established by ESC [6]. The Ethics Committee of the Hospital which approved the trial and all patients providedinformed consent to participate. Four of them withdrew consent for participation. Of the 95 patients who agreed to participate, 26 were classified as takotsubo syndrome, leaving 69 N-TT MINOCA patients. A study identification number was assigned to each patient and the recorded information was established and kept secure in the hospital.

MINOCA and N-TT MINOCA were compared with a group of 178 consecutive patients diagnosed with MI related to obstructive coronary arteries in the same hospital. Finally, a direct comparison of the psycho-emotional disorders was performed between the N-TT MINOCA subgroup and the takotsubo subgroup.

\section{Statistical analysis}

Continuous variables are presented as means and standard deviations. Categorical variables are provided with percentages. The Pearson $\chi^{2}$ test or Fisher exact test was used to compare categorical variables. A two-sided p value of less than 0.05 was considered statistically significant. Odds ratios are reported with $95 \%$ confidence intervals. Statistical software Epidat 3.1 was used (Consellería de Sanidade, Xunta de Galicia, Santiago de Compostela).

\section{Outcomes}

Data were obtained through interviews and review of medical records. Demographic and epidemiological data such as sex, age and traditional cardiovascular risk factors were registered (hypertension, dyslipidemia, diabetes or smoking). Psycho-emotional related characteristics like psychiatric illness (which needs or has needed, a specific medical treatment) or the recognition of self-perceived emotional stress (chronic and acute) were also recorded. Specific information about social aspects which are known to be stress modulating; including civil status, precarious work (defined as unstable employment or unemployment) or working at night shift were checked as well. Habits included the use of drugs that induce excitement (cocaine and amphetamines) [20] practising sports. Other characteristics analyzed were migraine and fibromyalgia. Standardized forms (EURO-CARDS) were used to collect patient data, including baseline characteristics, cardiovascular risk factors, coexisting illnesses, results of electrocardiography, echocardiography, coronary angiography and medication. All in-hospital complications and death from any cause were recorded.
Table 1. Pathophysiological mechanisms of myocardial infarction (MI) with non-obstructive coronary arteries.

\begin{tabular}{lc}
\hline Takotsubo syndrome & $26(27.4 \%)$ \\
Plaque disruption & $18(18.9 \%)$ \\
Suspected & 10 \\
Confirmed intracoronary imaging & 8 \\
Unknown mechanism & $11(11.6 \%)$ \\
Type II MI & $10(10.5 \%)$ \\
Myocarditis & $10(10.5 \%)$ \\
Coronary artery spasm & $8(8.4 \%)$ \\
Coronay emboli & $5(5.3 \%)$ \\
Coronary dissection & $3(3.2 \%)$ \\
Acute toxicity & $2(2.1 \%)$ \\
Coronary abnormalities & $1(1.1 \%)$ \\
Undiagnosed obstructive MI & $1(1.1 \%)$ \\
\hline
\end{tabular}

\section{Results}

From March 2015 to July 2017, 95 of 102 consecutive patients admitted to our hospital with a working diagnosis of MINOCA were included in the study. This represented $9.3 \%$ of all patients admitted with acute MI at that time. Table 1 shows the physiopathological mechanisms underlying the 95 patients included in the working diagnosis of MINOCA who were analyzed.

This group was compared with 178 consecutive patients admitted to the documented center during the previous year with acute MI who had obstructive coronary arteries on angiography.

Table 2 summarizes the main results. Patients with MINOCA, when compared with patients on the obstructive group were younger (61.7 vs. 66.0 years old, $\mathrm{p}=0.024$ ) and the proportion of females was higher ( $44.2 \%$ vs. $22.5 \%$, p < 0.01). They also had a better cardiovascular risk profile (diabetes: $13.7 \%$ vs. $39.5 \%, \mathrm{p}<0.001$, dyslipidemia: $34.7 \%$ vs. $61.4 \%, \mathrm{p}<0.001$; smoking: $48.4 \%$ vs. $64.9 \%$, $\mathrm{p}=0.012$; and hypertension: $52.6 \%$ vs. $63.6 \%$, $\mathrm{p}=0.09$ ).

The incidence of psychiatric illnesses was higher in the MINOCA group (29.7 vs. $12.9 \%$, $\mathrm{p}=0.001$ ). MINOCA patients recognized emotional stress in $75.7 \%$ of the cases, while only $32.1 \%$ of the obstructive related group did $(\mathrm{p}<0.001)$. It was acute in the $28.7 \%$ of MINOCA and in the $6.4 \%$ of obstructive related patients $(\mathrm{p}<0.001)$, and were referred to as chronic by $47.1 \%$ of the MINOCA, whereas $25.6 \%$ of obstructive related patients recognised chronic stress $(\mathrm{p}=0.002)$. 
Table 2. Main characteristics of MINOCA group compared with obstructive related AMI group.

\begin{tabular}{|c|c|c|c|}
\hline Characteristic & MINOCA $(n=95)$ & Obstructive related AMI $(n=178)$ & $\mathbf{P}$ \\
\hline Female sex & $42 / 95(44.2 \%)$ & $40 / 178(22.5 \%)$ & $<0.001$ \\
\hline Age [years] & $61.68 \pm 15.9$ & $66.0 \pm 13.3$ & 0.024 \\
\hline \multicolumn{4}{|l|}{ Traditional risk factors: } \\
\hline Hypertension & $50 / 95(52.6 \%)$ & $112 / 176(63.6 \%)$ & 0.09 \\
\hline Diabetes & $13 / 95(13.7 \%)$ & 70/177 (39.5\%) & $<0.001$ \\
\hline Dyslipidemia & $33 / 95(34.7 \%)$ & $108 / 176(61.4 \%)$ & $<0.001$ \\
\hline Smoking & $44 / 91(48.4 \%)$ & $11 / 171(64.9 \%)$ & 0.012 \\
\hline Coexisting medical condition: & $59 / 74(79.7 \%)$ & $66 / 158(41.8 \%)$ & $<0.001$ \\
\hline Psychiatric illnesses: & $28 / 94(29.7 \%)$ & $23 / 178(12.9 \%)$ & 0.001 \\
\hline Anxiety disorder & $12 / 94(12.8 \%)$ & $7 / 178(3.9 \%)$ & - \\
\hline Depression & $11 / 94(11.7 \%)$ & $13 / 178(7.3 \%)$ & - \\
\hline Bipolar disorder & $3 / 94(3.2 \%)$ & $0 / 178(0.0 \%)$ & - \\
\hline Schizophrenia & $2 / 94(2.1 \%)$ & $3 / 178(1.7 \%)$ & - \\
\hline Emotional stress: & $53 / 70(75.7 \%)$ & $50 / 156(32.1 \%)$ & $<0.001$ \\
\hline Acute & $20 / 70(28.7 \%)$ & $10 / 156(6.4 \%)$ & $<0.001$ \\
\hline Chronic & $33 / 70(47.1 \%)$ & $40 / 156(25.6 \%)$ & 0.002 \\
\hline Chronic headache & $10 / 94(10.6 \%)$ & $11 / 176(6.25 \%)$ & 0.24 \\
\hline Fibromyalgia & $1 / 94(1.1 \%)$ & $2 / 176(1.1 \%)$ & 1.00 \\
\hline \multicolumn{4}{|l|}{ Social aspects: } \\
\hline Married & $42 / 62(67.7 \%)$ & $106 / 149(71.1 \%)$ & 0.6 \\
\hline Single & $8 / 62(12.9 \%)$ & $9 / 149(6.0 \%)$ & 0.103 \\
\hline Divorced & $5 / 62(8.0 \%)$ & $13 / 149(8.7 \%)$ & 1.00 \\
\hline Precarious work & $9 / 25(36.0 \%)$ & $21 / 61(34.4 \%)$ & 1.00 \\
\hline Working at night shift & $21 / 54(38.9 \%)$ & $83 / 146(56.8 \%)$ & 0.027 \\
\hline Practicing sports & $27 / 88(30.6 \%)$ & $62 / 150(41.3 \%)$ & 0.13 \\
\hline Illegal exciting drugs use & $4 / 56(7.1 \%)$ & $1 / 157(0.6 \%)$ & 0.018 \\
\hline Combine external factors & $77 / 81(95.1 \%)$ & $129 / 135(95.6 \%)$ & 1.00 \\
\hline
\end{tabular}

AMI - acute myocardial infarction; MINOCA — myocardial infarction with non-obstructive coronary arteries

Regarding social aspects, paradoxically, the rate of working at night shift was higher in the obstructive group $(38.9 \%$ vs. $56.8 \%, \mathrm{p}=0.027)$. This could be explained due to smoking acting as a confounding factor, which was significantly higher in the night shift group $(78.2 \%$ vs. $37.2 \%$, $\mathrm{p}<0.001)$. The rate of precarious work was similar in both groups $(36.0 \%$ vs. $34.4 \%, \mathrm{p}=1.00)$. Neither the proportion of married people $(67.7 \%$ vs. $71.1, \mathrm{p}=0.6$ ) nor divorced (8.0 vs. 8.7, $\mathrm{p}=1.0$ ) showed differences between both groups, but there was a tendency of a higher rate of single people in the MINOCA group (12.9\% vs. $6.0 \%, \mathrm{p}=0.103)$. MINOCA patients consumed more excitement inducing drugs $(7.1 \%$ vs. $0.6 \%, \mathrm{p}=0.018)$ and tended to have a lower rate of people practicing sports $(30.6 \%$ vs. $41.3 \%, \mathrm{p}=0.13)$. There were no differences in the combined external factors (pre- carious work, working at night shift, illegal drugs use, single, divorced, non-practising sports) with a high proportion in both groups $95.1 \%$ vs. $95.6 \%$.

There were no differences in fibromyalgia, probably due to its low frequency (only 1 case in each group), neither in the prevalence of chronic headache $(10.6 \%$ vs. $6.25 \%, \mathrm{p}=0.24)$

\section{Non-takotsubo MINOCA}

The most prevalent pathophysiological mechanism in our patients was takotsubo syndrome, with 26 cases (27.4\% of MINOCA, $2.5 \%$ of all the acute $\mathrm{MI})$, leaving a sample of $69 \mathrm{~N}-\mathrm{TT}$ MINOCA patients.

Main results of subgroup analysis is shown in Table 3. Patients with N-TT MINOCA compared with patients in the obstructive group were younger and the proportion of females was higher. They also had a better cardiovascular risk profile. 
Table 3. Main characteristics of non-takosubo MINOCA group compared with obstructive related AMI group.

\begin{tabular}{|c|c|c|c|}
\hline Characteristic & $\begin{array}{l}\text { Non-takotsubo MINOCA } \\
\qquad(n=69)\end{array}$ & $\begin{array}{l}\text { Obstructive related AMI } \\
\qquad(\mathrm{n}=178)\end{array}$ & $\mathbf{P}$ \\
\hline Female sex & $22 / 69(31.9 \%)$ & $40 / 178(22.5 \%)$ & $<0.001$ \\
\hline Age [years] & $58.26 \pm 15.8$ & $66.0 \pm 13.3$ & $<0.001$ \\
\hline \multicolumn{4}{|l|}{ Traditional risk factors: } \\
\hline Hypertension & $31 / 69(44.9 \%)$ & $112 / 176(63.6 \%)$ & 0.09 \\
\hline Diabetes & $7 / 69(10.1 \%)$ & $70 / 177(39.5 \%)$ & $<0.001$ \\
\hline Dyslipidemia & $20 / 69(29.0 \%)$ & $108 / 176(61.4 \%)$ & $<0.001$ \\
\hline Smoking & $34 / 67(50.7 \%)$ & $11 / 171(64.9 \%)$ & 0.055 \\
\hline Coexisting medical condition: & $39 / 51(76.5 \%)$ & $66 / 158(41.8 \%)$ & $<0.001$ \\
\hline Psychiatric illnesses & $19 / 68(27.9 \%)$ & $23 / 178(12.9 \%)$ & 0.008 \\
\hline Emotional stress: & $34 / 48(70.8 \%)$ & $50 / 156(32.1 \%)$ & $<0.001$ \\
\hline Acute & $8 / 48(16.7 \%)$ & $10 / 156(6.4 \%)$ & 0.04 \\
\hline Chronic & $26 / 48(54.2 \%)$ & $40 / 156(25.6 \%)$ & $>0.001$ \\
\hline Chronic headache & $7 / 68(10.3 \%)$ & $11 / 176(6.25 \%)$ & 0.28 \\
\hline Fibromyalgia & $1 / 68(1.5 \%)$ & $2 / 176(1.1 \%)$ & 1.00 \\
\hline \multicolumn{4}{|l|}{ Social aspects: } \\
\hline Married & $30 / 42(71.4 \%)$ & $106 / 149(71.1 \%)$ & 1.00 \\
\hline Single & $6 / 42(14.3 \%)$ & $9 / 149(6.0 \%)$ & 0.102 \\
\hline Divorced & $4 / 42(9.5 \%)$ & $13 / 149(8.7 \%)$ & 1.00 \\
\hline Precarious work & $7 / 20(35.0 \%)$ & $21 / 61(34.4 \%)$ & 1.00 \\
\hline Working at night shift & $17 / 38(44.7 \%)$ & $83 / 146(56.8 \%)$ & 0.204 \\
\hline Practicing sports & $23 / 65(35.4 \%)$ & $62 / 150(41.3 \%)$ & 0.450 \\
\hline Illegal exciting drugs use & $4 / 38(10.5 \%)$ & $1 / 157(0.6 \%)$ & 0.005 \\
\hline Combine external factors & $56 / 59(94.9 \%)$ & $129 / 135(95.6 \%)$ & 1.00 \\
\hline
\end{tabular}

AMI - acute myocardial infarction; MINOCA - myocardial infarction with non-obstructive coronary arteries

The incidence of psychiatric illnesses was higher in the N-TT MINOCA group (27.9\% vs. $12.9 \%, \mathrm{p}<0.01)$. N-TT MINOCA patients recognized emotional stress in $70.8 \%$ of the cases, while only $32.1 \%$ of the obstructive related group $\operatorname{did}(\mathrm{p}<0.001)$.

Social habits also showed similar results in this MINOCA subgroup. N-TT MINOCA patients recognized as having a higher rate of taking stimulating drugs $(10.5 \%$ vs. $0.6 \%, \mathrm{p}=0.005)$. There was a tendency to a higher rate of single people in the MINOCA group (12.9\% vs. $6.0 \%, \mathrm{p}=0.103)$, with no differences in the other civil status. There were no differences in the rate of precarious work, nor working on night shift, nor practising sports. There were no differences in combined external factors, also with a high proportion in both groups $94.9 \%$ vs. $95.6 \%$. There were no differences in fibromyalgia ( $1.5 \%$ vs. $1.1 \%$ ) or with the proportion of chronic headache $(10.3 \%$ vs. $6.25 \%, \mathrm{p}=0.28)$ patients.
Psycho-emotional disorders in non-takotsubo MINOCA versus takotsubo syndrome

Table 4 summaries the results. There are no differences between N-TT MINOCA and takotsubo syndrome in the proportion of psychiatric disease ( $27.9 \%$ vs. $40.1 \%, \mathrm{p}=0.616)$ neither in the recognition of emotional stress $(70.8 \%$ vs. $86.4 \%$, $\mathrm{p}=0.232$ ). There was a significant difference when chronic and acute stress were divided, the acute stress was much higher in the takotsubo subgroup ( $16.7 \%$ vs. $77.3 \%, p=0.002)$. There were no differences in the proportion of chronic headache ( $10.3 \%$ vs. $11.5 \%)$ nor in the rate of fibromyalgia $(1.5 \%$ vs. $0.0 \%)$.

\section{Discussion}

This study is one of the first based on the definitions of the 2016 ESC Position Paper of MINOCA [6], with clear and reproducible inclusion criteria which has opened a new era of MINOCA research. 
Table 4. Main characteristics of non-takosubo myocardial infarction with non-obstructive coronary arteries (MINOCA) group compared with takotsubo group.

\begin{tabular}{lccc}
\hline Characteristic & Non-takotsubo MINOCA $(\mathbf{n}=69)$ & Takotsubo $(\mathbf{n}=\mathbf{2 6})$ & $\mathbf{P}$ \\
\hline Psychiatric illnesses & $19 / 68(27.9 \%)$ & $9 / 26(40.1 \%)$ & 0.616 \\
Emotional stress: & $34 / 48(70.8 \%)$ & $19 / 22(86.4 \%)$ & 0.232 \\
Acute & $8 / 48(16.7 \%)$ & $12 / 22(77.3 \%)$ & 0.002 \\
Chronic & $26 / 48(54.2 \%)$ & $7 / 22(31.8 \%)$ & 0.122 \\
Migraine & $7 / 68(10.3 \%)$ & $3 / 26(11.5 \%)$ & 1.00 \\
Fibromyalgia & $1 / 68(1.5 \%)$ & $0 / 26(0.0 \%)$ & 1.00 \\
\hline
\end{tabular}

Psychiatric illnesses and emotional stress were strongly associated with the risk of developing MINOCA. Social aspects which could have an impact on emotions did not show an association with MINOCA, even when combined. Only the use of drugs was related to MINOCA, and that could be explained due to a cause-effect relation more than through emotional modulation. MINOCA patients tended to be single, which is known to be an important emotional modulator. Taking the study as a whole, it shows that there is a high relationship between MINOCA and intrinsic psycho-emotional disorders, psychiatric diseases or the recognition of stress, whereas external factors which could act as stress modulating had no impact on the chance of developing MINOCA.

Previous studies have shown a relationship between takotsubo and psychiatric disorders [16], therefore it was excluded to verify the hypothesis that this relationship remains with the all MINOCA patients. The results of the present analysis of N-TT MINOCA are similar to those of the entire group, and in direct comparison, there were no differences in the proportion of psychiatric disease, nor in the recognition of emotional stress. Only the rate of acute stress was higher in takotsubo, which was already well established [16]. The results may be statistically significant utilizing a bigger number of patients, leaving a speculation that MINOCA patients have a higher rate of psycho-emotional disorders than those with obstructive related MI, and the proportion may even be higher in the takotsubo subgroup.

Thus, this study suggests that psycho-emotional disorders could be a risk factor for MINOCA. According to available research, this is the first evidence to link psycho-emotional disorders with MINOCA, which remained even in the N-TT MINOCA subgroup.

As it has been previously described [8-12], the present study shows that MINOCA patients are younger, with a higher proportion of women and have a better cardiovascular risk profile. A higher proportion of chronic headache in the MINOCA group was not found which was shown to be the case by other authors [21].

The relation between MI and psychiatric disorders has already been described $[22,23]$ but it is also known that psychiatric patients have higher chance of cardiovascular risk factors [24], unlike what was shown in this study, MINOCA patients had a better cardiovascular profile. Recently, a study suggested that optimistic patients have a better prognosis after MI [25], while others linked heart disease with a worse outlook [26, 27]. This concurs with the present hypothesis that negative emotions could be a risk factor for MINOCA.

These findings could be explained by the impact of emotions on adrenergic regulation and its relationship with endothelium function [28], which is speculated to be one of the pathophysiological mechanisms responsible and underlying MINOCA.

If these findings are confirmed, on one hand, it could be an indicator to general practitioners on the importance of primary prevention in high-risk patients and on the other hand, develop a new target for secondary prevention [29]. One application could include management of psycho-emotional disorders in cardiac rehabilitation programs for these patients. Nevertheless, further research is necessary to bring definitive evidence to this field.

\section{Limitations of the study}

This study has some limitations. There was no search for undiagnosed psychiatric disease; a Mini International Neuropsychiatry Interview [30] would be a practical way of screening. Another limitation was that patients were asked for recognition of emotional stress in a stressful situation, which could have led to an increase in positive answers.

\section{Conclusions}

Psycho-emotional disorders are related with MINOCA. The presence of psychiatric illnesses 
was higher in MINOCA than in MI with obstructive coronary arteries group $(29.7 \%$ vs. $12.9 \%$, $\mathrm{p}=0.001$ ), and MINOCA patients recognized emotional stress in $75.7 \%$ of the cases, while only $32.1 \%$ of the obstructive related group did $(\mathrm{p}<0.001)$. The relationship remained after excluding takotsubo from the analysis in psychiatric diseases $(27.9 \%$ vs. $12.9 \%, \mathrm{p}<0.01)$ and in the recognition of emotional stress $(70.8 \%$ vs. $32.1 \%, \mathrm{p}<0.001)$. Social habits which could act as stress modulating showed no relation with MINOCA.

\section{Acknowledgements}

This work was supported by unrestricted grants from Red Tematica de Investigacion Cooperativa en Enfermedades Cardivasculares (RIC) RD12/0042/0067 of the Instituto de Salud Carlos III (Ministerio de Economia y Competitividad), and by a competitive grant from Section of Clinical Cardiology of the Spanish Society of Cardiology.

\section{Conflict of interest: None declared}

\section{References}

1. Diver DJ, Bier JD, Ferreira PE, et al. Clinical and arteriographic characterization of patients with unstable angina without critical coronary arterial narrowing (from the TIMI-IIIA Trial). Am J Cardiol. 1994; 74(6): 531-537, doi: 10.1016/0002-9149(94)90739-0, indexed in Pubmed: 8074033.

2. Pasupathy S, Air T, Dreyer RP, et al. Systematic review of patients presenting with suspected myocardial infarction and nonobstructive coronary arteries. Circulation. 2015; 131(10): 861-870, doi: 10.1161/ CIRCULATIONAHA.114.011201, indexed in Pubmed: 25587100.

3. Libby P. Current concepts of the pathogenesis of the acute coronary syndromes. Circulation. 2001; 104(3): 365-372, doi: 10.1161/01.cir.104.3.365, indexed in Pubmed: 11457759.

4. Gehrie ER, Reynolds HR, Chen AY, et al. Characterization and outcomes of women and men with non-ST-segment elevation myocardial infarction and nonobstructive coronary artery disease: results from the Can Rapid Risk Stratification of Unstable Angina Patients Suppress Adverse Outcomes with Early Implementation of the ACC/AHA Guidelines (CRUSADE) quality improvement initiative. Am Heart J. 2009; 158(4): 688-694, doi: 10.1016/j.ahj.2009.08.004, indexed in Pubmed: 19781432.

5. Kang WYu, Jeong MHo, Ahn YK, et al. Korea Acute Myocardial Infarction Registry Investigators. Are patients with angiographically near-normal coronary arteries who present as acute myocardial infarction actually safe? Int J Cardiol. 2011; 146(2): 207-212, doi: 10.1016/j.jicard.2009.07.001, indexed in Pubmed: 19664828.

6. Agewall S, Beltrame JF, Reynolds HR, et al. WG on Cardiovascular Pharmacotherapy. ESC working group position paper on myocardial infarction with non-obstructive coronary arteries. Eur Heart J. 2017; 38(3): 143-153, doi: 10.1093/eurheartj/ehw149, indexed in Pubmed: 28158518.

7. Barrabés J, Bardají A, Jiménez-Candil J, et al. Prognosis and Management of Acute Coronary Syndrome in Spain in 2012: The DIOCLES Study. Rev Esp Cardiol (Engl Ed). 2015; 68(2): 98-106, doi: 10.1016/j.rec.2014.03.010, indexed in Pubmed: 25623429.
8. Pasupathy S, Air T, Dreyer RP, et al. Systematic review of patients presenting with suspected myocardial infarction and nonobstructive coronary arteries. Circulation. 2015; 131(10): 861-870, doi: 10.1161/CIRCULATIONAHA.114.011201, indexed in Pubmed: 25587100.

9. Kardasz I, De Caterina R. Myocardial infarction with normal coronary arteries: a conundrum with multiple aetiologies and variable prognosis: an update. J Intern Med. 2007; 261(4): 330-348, doi: 10.1111/j.1365-2796.2007.01788.x, indexed in Pubmed: 17391108.

10. Papanicolaou M, Califf R, Hlatky M, et al. Prognostic implications of angiographically normal and insignificantly narrowed coronary arteries. The American Journal of Cardiology. 1986; 58(13): 1181-1187, doi: 10.1016/0002-9149(86)90378-4.

11. Rossini R, Capodanno D, Lettieri C. Long-Term Outcomes of Patients With Acute Coronary Syndrome and Non-obstructive Coronary Artery Disease. Am J Cardiol. 2013; 112: 150e155.

12. Patel MR, Chen AY, Peterson ED, et al. Prevalence, predictors, and outcomes of patients with non-ST-segment elevation myocardial infarction and insignificant coronary artery disease: results from the Can Rapid risk stratification of Unstable angina patients Suppress ADverse outcomes with Early implementation of the ACC/AHA Guidelines (CRUSADE) initiative. Am Heart J. 2006; 152(4): 641-647, doi: 10.1016/j.ahj.2006.02.035, indexed in Pubmed: 16996828.

13. Borja Ibanez, Stefan James, Stefan Agewall, et al. 2017 ESC Guidelines for the management of acute myocardial infarction in patients presenting with ST-segment elevation: The Task Force for the management of acute myocardial infarction in patients presenting with ST-segment elevation of the European Society of Cardiology (ESC), European Heart Journal, hx393. https://doi. org/10.1093/eurheartj/ehx393.

14. Daniel M, Ekenbäck C, Agewall S, et al. Risk Factors and Markers for Acute Myocardial Infarction With Angiographically Normal Coronary Arteries. Am J Cardiol. 2015; 116(6): 838-844, doi: 10.1016/j.amjcard.2015.06.011, indexed in Pubmed: 26251000.

15. Pais J, Izquierdo B, González V, et al. Incidence, clinical profile and prognosis of patients with myocardial infarction with nonobstructive coronary arteries in the real world. J Am Coll Cardiol. 2017; 69(11): 142, doi: 10.1016/s0735-1097(17)33531-3.

16. Templin C, Ghadri JR, Diekmann J, et al. on behalf of InterTAK investigators. Clinical Features and Outcomes of Takotsubo (Stress) Cardiomyopathy. N Engl J Med. 2015; 373: 929-38.

17. Thygesen K, Alpert JS, Jaffe AS, et al. Joint ESC/ACCF/AHA/ /WHF Task Force for Universal Definition of Myocardial Infarction, Authors/Task Force Members Chairpersons, Biomarker Subcommittee, ECG Subcommittee, Imaging Subcommittee, Classification Subcommittee, Intervention Subcommittee, Trials \& Registries Subcommittee, Trials \& Registries Subcommittee, Trials \& Registries Subcommittee, Trials \& Registries Subcommittee, ESC Committee for Practice Guidelines (CPG), Document Reviewers, Writing Group on the Joint ESC/ACCF/ AHA/WHF Task Force for the Universal Definition of Myocardial Infarction, ESC Committee for Practice Guidelines (CPG). Third universal definition of myocardial infarction. Eur Heart J. 2012; 33(20): 2551-2567, doi: 10.1093/eurheartj/ehs184, indexed in Pubmed: 22922414.

18. Scanlon PJ, Faxon DP, Audet AM, et al. ACC/AHA guidelines for coronary angiography. A report of the American College of Cardiology/American Heart Association Task Force on practice guidelines (Committee on Coronary Angiography). Developed in 
collaboration with the Society for Cardiac Angiography and Interventions. J Am Coll Cardiol. 1999; 33(6): 1756-1824, indexed in Pubmed: 10334456.

19. Prasad A, Lerman A, Rihal CS. Apical ballooning syndrome (Tako-Tsubo or stress cardiomyopathy): a mimic of acute myocardial infarction. Am Heart J. 2008; 155(3): 408-417, doi: 10.1016/j. ahj.2007.11.008, indexed in Pubmed: 18294473.

20. Sinha R. Chronic stress, drug use, and vulnerability to addiction. Ann N Y Acad Sci. 2008; 1141: 105-130, doi: 10.1196/annals.1441.030, indexed in Pubmed: 18991954.

21. Raymond R, Lynch J, Underwood D, et al. Myocardial infarction and normal coronary arteriography: a 10 year clinical and risk analysis of 74 patients. J Am Coll Cardiol. 1988; 11(3): 471-477, doi: 10.1016/0735-1097(88)91519-7, indexed in Pubmed: 3278033.

22. Harris EC, Barraclough B. Excess mortality of mental disorder. Br J Psychiatry. 1998; 173: 11-53, doi: 10.1192/bjp.173.1.11, indexed in Pubmed: 9850203.

23. Lett HS, Blumenthal JA, Babyak MA, et al. Depression as a risk factor for coronary artery disease: evidence, mechanisms, and treatment. Psychosom Med. 2004; 66(3): 305-315, doi: 10.1097/00006842-200405000-00004, indexed in Pubmed: 15184688 .

24. Casey DE. Metabolic issues and cardiovascular disease in patients with psychiatric disorders. Am J Med. 2005; 118 Suppl 2: 15S-22S, doi: 10.1016/j.amjmed.2005.01.046, indexed in Pubmed: 15903291.
25. Weiss-Faratci N, Lurie I, Benyamini Y, et al. Optimism During Hospitalization for First Acute Myocardial Infarction and LongTerm Mortality Risk: A Prospective Cohort Study. Mayo Clin Proc. 2017; 92(1): 49-56, doi: 10.1016/j.mayocp.2016.09.014, indexed in Pubmed: 27876316.

26. Roest AM, Heideveld A, Martens EJ, et al. Symptom dimensions of anxiety following myocardial infarction: associations with depressive symptoms and prognosis. Health Psychol. 2014; 33(12): 1468-1476, doi: 10.1037/a0034806, indexed in Pubmed: 24274800

27. Gorkin L, Schron EB, Brooks MM, et al. Psychosocial predictors of mortality in the Cardiac Arrhythmia Suppression Trial-1 (CAST-1). Am J Cardiol. 1993; 71(4): 263-267, doi: 10.1016/00029149(93)90788-e, indexed in Pubmed: 8427165.

28. Ghiadoni L, Donald AE, Cropley M, et al. Mental stress induces transient endothelial dysfunction in humans. Circulation. 2000; 102(20): 2473-2478, indexed in Pubmed: 11076819.

29. Lindahl B, Baron T, Erlinge D, et al. Medical therapy for secondary prevention and long-term outcome in patients with myocardial infarction with nonobstructive coronary artery disease. Circulation. 2017; 135(16): 1481-1489, doi: 10.1161/CIRCULATIONAHA.116.026336, indexed in Pubmed: 28179398.

30. Lecrubier Y, Sheehan DV, Weiller E, et al. The Mini International Neuropsychiatric Interview (MINI). A short diagnostic structured interview: reliability and validity according to the CIDI. Eur Psychiatry. 1997; 12(5): 224-231, doi: 10.1016/s0924-9338(97)83296-8. 